\section{Rheumatologen assistieren Onkologen}

Für die Tumorerkrankung seien irAE wohl ein positives Signal. So hätten $65 \%$ von 551 Patienten mit kompletter oder partieller Remission unter CPi irAE gehabt, aber nur $24 \%$ mit Tumorprogress [2]. Studien zeigten einen „dramatischen, signifikanten Unterschied" im progressionsfreien Überleben für Patienten mit irAE $[2,3]$.

》) Als Indikation, die Immuntherapie doch zu beenden, nennt die EULAR speziell schwere Myositiden

So sieht Schulze-Koops die Rheumatologen auch in der Pflicht, den Onkologen zu assistieren und die Autoimmunphänomene „in den Griff zu kriegen" - ohne die überlebenswichtige onkologische Therapie in Frage zu stellen. Als Mitherausgeber der EULAR-Empfehlungen zu Diagnose und Management von irAE unter CPi [4] schlägt er nach NSAR zunächst lokale, wenn nötig systemische Glukokortikoide, möglichst $<10 \mathrm{mg} / \mathrm{d}$ Prednison vor, anschließend csDMARDS wie MTX, Ciclosporin A oder Leflunomid.

"Bei Methotrexat haben wir mittlerweile keine Sorge in den Dosierungen, die wir in der Rheumatologie einsetzen", es gebe keine Kontraindikationen für die CPi. Falls erforderlich, könnten Biologika verabreicht werden: Abatacept, das selbst an CTLA angreift, würde er "eher ganz am Ende" überlegen, aber TNFalpha- oder IL-6-Inhibitoren hätten möglicherweise sogar einen positiven Effekt auf die Tumortherapie. Als Indikation, die Immuntherapie doch zu beenden, nennen die EULAR-Empfehlungen speziell schwere Myositiden. Schulze-Koops: „Es ist die lebensbedrohliche Erkrankung, die behandelt werden muss."

\section{Literatur}

1. Cappelli LC et al (2017) Immune-related adverse effects of cancer immunotherapy implications for rheumatology. Rheum Dis Clin North Am 43(1):65-78
2. Kostine M et al (2018) OP0088 Immunerelated adverse events of cancer immunotherapy - when inflammatory side effects are associated with survival: a single-centre prospective cohort study. Ann Rheum Dis 77(Suppl. 2):94-95

3. Tison A et al (2019) Safety and efficacy of immune checkpoint inhibitors in patients with cancer and preexisting autoimmune diseases: a nationwide multicenter retrospective study, Ann Rheum Dis 71(12):2100-2111

4. Kostine $\mathrm{M}$ et al (2020) EULAR points to consider for the diagnosis and management of rheumatic immune-related adverse events due to cancer immunotherapy with checkpoint inhibitors. Ann Rheum Dis Published Online First: 23 April 2020. https://doi.org/ 10.1136/annrheumdis-2020-217139

Hinweis des Verlags. Der Verlag bleibt in Hinblick auf geografische Zuordnungen und Gebietsbezeichnungen in veröffentlichten Karten und Institutsadressen neutral.

rheuma plus $2021 \cdot 20: 66-67$ https://doi.org/10.1007/s12688-02100402-5

(C) Springer-Verlag GmbH Austria, ein Teil von Springer Nature 2021
Quelle: Dr.

Bianca Bach,

SpringerMedizin.de (basierend auf der Session CheckpointInhibitoren. 48. Kongress der DGRh, München (virtuell), 9.-12.09.2020)

\title{
Rot um die Augen nach Corona-Infektion: ein Alarmsignal?
}

Eine Multisystembeteiligung im Sinne eines MIS-C gilt als äußerst seltene Komplikation einer SARS-CoV-2-Infektion beim Kind. Daran denken sollte man zum Beispiel, wenn der kleine Patient neben unklarem Fieber bestimmte Symptome rund um die Augen präsentiert.

Seit Frühjahr 2020 wird immer wieder von ungewöhnlichen Verlaufsformen einer SARS-CoV-2-Infektion bei Kindern berichtet, die mit deutlich erhöhten Entzündungsmarkern und einer Multisystembeteiligung, ähnlich dem Kawasaki-Syndrom (KS), einhergehen. Dieses von der CDC (Centers for Disease Control and Prevention) mittlerweile als Multisystem Inflammatory Syndrome in Children (MIS-C) bezeichnete Krankheitsbild ist mit einer Inzidenz von etwa 2 von 100.000 SARS-CoV-2-Infizierten unter 21 Jahren äußertselten; aufgrund des oft schweren Verlaufs ist jedoch eine möglichst frühzeitige Diagnostik und eventuell auch die Abgrenzung vom KS entscheidend.

》) MIS-C geht in vielen Fällen mit mukokutanen Zeichen einher

Helfen könnte dabei die Beobachtung, dass das MIS-C in vielen Fällen (Stu- dien zufolge in bis zu $74 \%$ ) mit mukokutanen Zeichen einhergeht. Diese zu charakterisieren, hat sich ein Team der New York University Grossman School of Medicine vorgenommen [1].

\section{Befunde an Haut und Schleim-} häuten bei über $80 \%$

In der retrospektiven Fallserie von Trevor K. Young und Kollegen lag die Inzidenz mukokutaner Befunde bei den insgesamt 35 Kindern mit bestä- 
tigtem oder vermutetem MIS-C bei $83 \%$. Die Veränderungen an Haut- und Schleimhäuten waren vielgestaltig, wobei einige besonders häufig auffielen, darunter

- konjunktivale Injektion (21 Fälle),

- Erythem der Handflächen und Fußsohlen (18),

- Hyperämie der Lippen (17) bzw. aufgesprungene Lippen (13),

- Erdbeerzunge (8),

- periorbitales Erythem und Ödem (7) und

- Liderythem (6).

Seltener traten masern- oder scharlachartige, urtikarielle oder netzförmige Hautausschläge auf, die Lokalisation war dabei sehr unterschiedlich. Kinder unter beziehungsweise über drei Jahre waren insgesamt etwa gleich häufig betroffen. Zu erwähnen ist, dass es sich zu etwa einem Drittel um dunkelhäutige Kinder handelte, ein weiteres Drittel war hispanischen Ursprungs.

\section{Keine Assoziation mit Krankheits-} schwere

Mit der Krankheitsschwere schien keine Assoziation zu bestehen. So mussten Kinder mit den beschriebenen Hautveränderungen nicht etwa häufiger in die Intensivstation und benötigten auch nicht häufiger eine inotrope Medikation aufgrund einer Herzbeteiligung.

》) Die Dauer der mukokutanen Zeichen war höchst unterschiedlich
Etwa $60 \%$ der kleinen Patienten erfüllten Kriterien, die klassischerweise mit einem KS in Zusammenhang gebracht werden, so Young et al. Dazu gehört zum Beispiel die Erdbeerzunge. Dagegen sei zum Beispiel das periorbitale Ödem beziehungsweise Erythem, welches an eine Lichtdermatose erinnere, eher untypisch für ein $\mathrm{KS}$, ebenso wie die Lidrötung und der retikuläre Hautausschlag, der an ein Erythema infectiosum erinnere.

\) Abgesehen von den Hautzeichen war bei den meisten Kindern der Magen-Darm-Trakt betroffen

Fieber hatten insgesamt 29 Kinder mit MIS-C entwickelt, davon 19 vor Auftreten der ersten Hautzeichen. Im Schnitt hielt das Fieber knapp drei Tage an. Bei den mukokutanen Zeichen war die Dauer höchst unterschiedlich und reichte von wenigen Stunden bis zu elf Tagen (im Mittel fünf Tage).

\) Die D-Dimer-Spiegel waren in nahezu allen Fällen erhöht

In neun Fällen hatten sich bei der Nachbeobachtung an Hand- und Fußsohlen Desquamationen entwickelt, wobei dies bis zu einem Monat nach Fieberbeginn gedauert haben konnte. Nur in einem Fall war aus dem morbilliformen Hautausschlag eine Biopsie entnommen worden; bei der histologischen Untersuchung zeigte sich ein unspezifisches perivaskuläres lymphozytäres Infiltrat.

\section{Das Wichtigste in Kürze}

Gibt es Befunde an Haut und Schleimhaut, die beim fiebernden Kind nach Ausschluss anderer Ursachen auf den Beginn eines MIS-C hinweisen?

In einer Fallserie aus New York waren vor allem folgende Befunde mit einem MIS-C assoziiert: konjunktivale Injektion, palmar-plantares Erythem, hyperämisierte Lippen, Periorbitalerythem/-ödem, Erdbeerzunge und Liderythem.

Bedeutung: Während einige Befunde sehr stark mit dem Kawasaki-Syndrom überlappen, scheinen andere, vor allem das Liderythem und das periorbitale Ödem, eher mit dem MIS-C in Zusammenhang zu stehen.

Einschränkung: Retrospektive Fallserie, positiver SARS-CoV-2-Test lag nicht in allen Fällen vor.

\section{Respiratorische Symptome eher} selten

Abgesehen von den Hautzeichen war bei den meisten Kindern der MagenDarm-Trakt betroffen. Respiratorische Symptome wie Husten oder Schnupfen berichteten nur fünf. Die D-DimerSpiegel waren in nahezu allen Fällen erhöht, wenngleich thromboembolische Komplikationen ausblieben. Bei immerhin 19 Kindern fanden sich ein erhöhtes Troponin und/oder erhöhte BNP-Spiegel, was für eine Herzbeteiligung spricht. Letztlich konnten jedoch alle Kinder in gutem Zustand nach Hause entlassen werden.

\) Veränderungen an Haut und Schleimhaut sind bei fiebernden Kindern ernst zu nehmen

Die Autoren weisen darauf hin, dass bei zehn der allesamt stationär behandelten Kinder kein positiver Test auf SARS-CoV-2 vorlag. Dies könnte mit der zeitlichen Verzögerung zusammenhängen, mit der die Kinder vorgestellt wurden.

\section{Das Fazit der New Yorker Forscher} Veränderungen an Haut und Schleimhaut sind bei fiebernden Kindern ernst zu nehmen und könnten in Zeiten der Corona-Pandemie durchaus auf ein MIS-C hinweisen, wenngleich sie für dieses Krankheitsbild alles andere als spezifisch sind.

\section{Literatur}

1. Young TK et al (2020) Mucocutaneous Manifestations of Multisystem Inflammatory Syndrome in Children During the COVID-19 Pandemic. JAMA Dermatol. https://doi.org/ 10.1001/jamadermatol.2020.4779

Hinweis des Verlags. Der Verlag bleibt in Hinblick auf geografische Zuordnungen und Gebietsbezeichnungen in veröffentlichten Karten und Institutsadressen neutral.

rheuma plus $2021 \cdot 20: 67-68$ https://doi.org/10.1007/s12688-02100423-0

(c) Springer-Verlag GmbH Austria, ein Teil von Springer Nature 2021 